\title{
DISCLAIMER
}

This report was prepared as an sccount of work sponsored by an agency of the Unitod States Government. Neither the United Stales Govetnment nor any ageney :hercol, nor any of their employees, makes any warranty, express or implied, or assumes any legal liability of responsibility for the accuracy, completeness, or usefulness of any information, apparatus, produch, or process disclosed, or represents that its use would not infringe privately owned rights. Reference herein to any specific commercial product, process, or service by trade name, trademark. manufacturet, or otherwise does not necessarily constitute or imply its endorsement, recommendation, or favoring by the United States Government or any ageacy thereof. The views and opinions of authors expressed herein do not necessarily state or reflect those of the United States Governmept or any agency thereof.

\section{MODELING OF THE SAWTOOTH INSTABILITY IN TOKAMAKE USING A CURRENT VISCOSITY TERM}

PPPL- 2547

DE88 015825

\author{
D.J. Ward and S.C. Jardin \\ Plasma Physics Laboratory, Priaceton University \\ Princeton, New Jersey 08543
}

\begin{abstract}
We propose a new method for modeling the sawtooth instability and other MHD activity in axisymmetric tolamak transport simulations. A hyper-resistivity (or current viscosity) term is included in the mean field Ohm's law to describe the effects of the three-dimensional fluctuating fields on the evolution of the inverse transform, $q$, characterizing the mean fields. This term has the effect of flattening the current profile, while dissipating energy and conserving helicity. A fully implicit MHD transport and 2-D toroidal equilibrium code has been developed to calculate the evolution in time of the $q$-profile and the current profile using this new term. The results of this code are compared to the Kadomtsev reconnection model in the circular cylindrical limit.
\end{abstract}




\section{INTRODUCTION}

Tokamak transport simulation codes presently play an active and important role in magnetic fusion research. These codes have proved useful not as methods for predicting plasma behavior from first principles, but rather as lower dimensional reduced descriptions which enforce the appropriate conservation equations. They also account for fine scale or fluctuating three-dimensional phenomena by means of 'anomalous' coefficients or heuristically motivated prescriptions.

One such class of phenomena which is easential to model at some level is the sawtooth instability [1]. There are presently several approaches used in doing this. One approsch [2] is to incorporate the Kadomtsev complete reconnection model [3] at time intervals which are either predetermined or are linked in some way to the plasma evolution. Another method $[4,5]$ used is to flatten the resistivity profile in the interior of the magnetic surface which has $q=1$.

Each of these prescriptions has some merit, but each also has some inconsistencies. The Kadomtsev model is only stijctly applicable to a cylindrical plasma of circular cross section where a pure helical mode can exist. Even then, there is no guarantee that the helical structure would fully reconnect as assumed by this model. The resistivity flattening model does not explicitly conserve magnetic helicity as would be done by the underlying MHD instability.

This paper deals with a new method for modeling the sawtooth instability in a tokamak transport code, based on introducing a hyper-resistivity or current viscosity term in the transport equations. It has been shown by several authors [6-9] that the effects of threedimensional MHD fluctuations on the force-free mean field of a plasma can be represented by the introduction of a new term with one free parameter into Ohm's law such that

$$
\vec{E}+\vec{v} \times \vec{B}=\eta_{\|} \vec{J}_{\|}+\eta_{\perp} \vec{J}_{\perp}-\frac{\vec{B}}{B^{2}} \vec{\nabla} \cdot\left\{\left[\lambda \vec{\nabla}\left(\frac{\vec{J} \cdot \vec{B}}{B^{2}}\right)\right]-\left[\vec{u} \times \vec{\nabla}\left(\frac{\vec{J} \cdot \vec{B}}{B^{2}}\right)\right]\right\}
$$

where $\lambda$ is an arbitrary, positive function of position, and where $\vec{u}$ is an arbitrary vector function of position that may be chosen arcosding to mathematical convenience. The form of the function $\lambda$ has been calculated explicitly for tearing modes in the reversed-field pinch (RFP) $[10,11]$ where hyper-resistivity is shown to sustain the magnetic field reversal and to produce plasmas near the force-free Taylor state $[10,12]$. Here, however, we make use only of the fact that an arbitrary, positive $\lambda$ follows from the derivation in Ref. [9].

The new term conserves magnetic helicity. Equation (1) is, in fact, the unique form of a force-free mean field. Thm's law under the assumptions [9]: (1) the exact magnetic field energy and helicity are closely approximated by the energy and helicity of the mean field, (2) the fluctuating field can cause differential transport of both the field energy and helicity, and (3) the fluctuating field can lead to enhanced dissipation of field energy but 
not of field helicity. The underlying physical instabilities leading to sawtooth oscillations should satisfy these assumptions; therefore we propose to model sawteeth and other 3-D MHD phenomena using this equation in such an MHD transport model.

\section{THEORY}

It can be shown [13] that in a toroidal system with nested magnetic surfaces, the $q$ profile (safety factor profile) develops in time according to

$$
\frac{\partial}{\partial t} q^{-1}=\frac{d}{d \Phi} V_{L}
$$

where $\Phi$ is the toroidal flux contained inside the $\Phi=$ constant surface, and $V_{L}(\Phi)$ is the loop voltage, defined by

$$
V_{L}=\frac{2 \pi\langle\mathcal{J} \vec{B} \cdot \vec{R}\rangle}{\langle J \vec{B} \cdot \vec{\nabla} \phi\rangle}
$$

Angle brackets denote the flux surface average, defined by

$$
<a>=\frac{1}{2 \pi} \int_{0}^{2 \pi} a d \theta \text {. }
$$

Here $\vec{R}$ represents the right-hand side of Eq. (1), which can be written

$$
\vec{E}+\vec{v} \times \vec{B}=\vec{R}=\vec{R}_{C}+\vec{R}_{H},
$$

and the Jacobian, $\mathcal{J}$, of the $(\Phi, \theta, \phi)$ magnetic flux coordinate system is defined by

$$
\mathcal{J}=[\vec{\nabla} \Phi \times \vec{\nabla} \theta \cdot \vec{\nabla} \phi]^{-1} .
$$

Here $\phi$ represents the axisymmetric toroidal angle with $|\nabla \phi|^{2}=R^{-2}, \theta$ is the poloidal angle, and $(R, \phi, Z)$ are conventional cylindrical coordinates.

If we first consider only the classical term, $\vec{R}_{C}=\eta_{\|} \vec{J}_{\|}+\eta_{\perp} \vec{J}_{\perp}$, the loop voltage appearing in Eq. (2) is

$$
V_{L}^{\text {class }}=2 \pi \eta_{\|} \frac{\langle\mathcal{J} \vec{J} \cdot \bar{B}\rangle}{\langle J \bar{B} \cdot \vec{\nabla} \phi\rangle}=\frac{2 \pi \eta_{\|}(g(\Phi))^{2}}{\mu_{0}} \frac{d}{d \Phi}\left(\frac{\left\langle|\nabla \Phi|^{2} \frac{J}{R^{2}}\right\rangle}{g(\Phi)} q^{-1}\right) .
$$

We have taken the axisymmetric (or mean) magnetic field to be represented in the usual way, 


$$
\vec{B}=\vec{\nabla} \phi \times \vec{\nabla} \Psi+g(\Phi) \vec{\nabla} \phi,
$$

with $\Psi$ being the poloidal magnetic flux per radian, $\vec{\nabla} \Psi=(2 \pi q)^{-1} \vec{\nabla} \Phi$, and $g$ the toroidal field function, $g(\Phi)=\left[2 \pi<\frac{g}{R^{2}}>\right]^{-1}$.

Next consider the new term in the mean field Ohm's law that describes the effects of the 3-D turbulent field. The hyper-resistivity term is

$$
\vec{R}_{H}=-\frac{\vec{B}}{B^{2}} \vec{\nabla} \cdot[(\lambda \vec{\nabla} \sigma)-(\vec{u} \times \vec{\nabla} \sigma)],
$$

where,

$$
\sigma=\frac{\vec{J} \cdot \vec{B}}{B^{2}}=\frac{g^{2}(\Phi)}{\mu_{0} B^{2} J}\left\{\frac{\partial}{\partial \Phi}\left[\frac{\mathcal{J}|\nabla \Phi|^{2}}{R^{2}} \frac{1}{g(\Phi)} \Psi^{\prime}\right]+\frac{\partial}{\partial \theta}\left[\frac{\mathcal{J}(\vec{\nabla} \theta \cdot \vec{\nabla} \Phi)}{\vec{R}^{2}} \frac{1}{g(\Phi)} \Psi^{\prime}\right]\right\}
$$

the prime denoting differentiation with respect to $\Phi$.

We take advantage of the fact that $\vec{u}$ can be any arbitrary function of position, and specify the form for $\vec{u}$ such that the terms with theta derivatives of $\sigma$ cancel, thus

$$
\vec{u}=-\lambda \mathcal{J}(\vec{\nabla} \Phi \cdot \vec{\nabla} \theta) \vec{\nabla} \phi .
$$

The loop voltage corresponding to the hyper-resistivity term, Eq. (6), then becomes

$$
V_{L}^{H}=2 \pi \frac{\left\langle\mathcal{J} \vec{B} \cdot \vec{R}_{H}\right\rangle}{\langle\mathcal{J} \vec{B} \cdot \vec{\nabla} \phi\rangle}=-(2 \pi)^{2} \frac{\partial}{\partial \Phi}\left\langle\lambda \mathcal{J}|\nabla \Phi|^{2} \frac{\partial \sigma}{\partial \Phi}\right\rangle .
$$

Considerable simplification can be obtained by restricting the form of $\lambda$, the arbitrary function of position appearing in Eq. (1), to be of the form

$$
\lambda=\frac{\Lambda(\Phi)}{(2 \pi)^{2} \mathcal{J}|\nabla \Phi|^{2}},
$$

where $\Lambda(\Phi)$ is an arbitrary function of toroidal flux. In addition, we choose the angle $\theta$ in the $(\Phi, \theta, \phi)$ flux coordinate system so that the Jacobian, $\mathcal{J}$, is given by the Boozer form [14]

$$
\mathcal{J}=\frac{f(\Phi)}{\mu_{0} B^{2}}
$$

where

$$
f(\Phi) \equiv \frac{\mu_{0}}{(2 \pi)^{2}<B^{-2}>} \frac{d V}{d \Phi},
$$


$V(\Phi)$ being the volume enclosed by the flux surface with label $\Phi$.

Including the loop voltage due to the classical term, Eq. (4), and that due to the hyperresistivity term, Eq. (9), in the time evolution equation for $q$, Eq. (2), gives the final form for the complete equation to advance $q$ in time relative to surfaces of constant toroidal flux

$$
\begin{aligned}
& \frac{\partial}{\partial t} q^{-1}=\frac{d}{d \Phi}\left[\frac{2 \pi \eta_{\|}(\Phi)}{\mu_{0}}(g(\Phi))^{2} \frac{d}{d \Phi}\left(\frac{\left\langle|\nabla \Phi|^{2} \frac{J}{R^{2}}\right\rangle}{g(\Phi)} q^{-1}\right)\right] \\
& -\frac{d^{2}}{d \Phi^{2}}\left\{\left(\Lambda(\Phi) \frac{d}{d \Phi}\left(\frac{g^{2}(\Phi)}{f(\Phi)} \frac{d}{d \Phi}\left[\frac{\left\langle|\nabla \Phi|^{2} \frac{J}{R^{2}}\right\rangle}{g(\Phi)} q^{-1}\right]\right)\right)\right\} .
\end{aligned}
$$

Equation (11) includes classical resistive field diffusion, as well as the new term representing the effect of the 3-D turbulent fields. We model sawteeth with this equation by evolving the q-profile in time with the resistive term only. When some threshold is reached (e.g., $q_{0}$ reaches some minimum value), we 'trigger' the sawtooth by turning on the hyper-resistivity term within some inner radius of the cross section.

As a first test of this model we consider the large aspect ratio cylindrical tokamak limit. The evolution equation, Eq. (11), becomes the simpler 1-D equation:

$$
\frac{\partial \Psi}{\partial t}=\frac{\eta(r)}{\mu_{0}} \frac{1}{r} \frac{\partial}{\partial r}\left(r \frac{\partial \Psi}{\partial r}\right)-\frac{1}{r} \frac{\partial}{\partial r}(\bar{\lambda} r) \frac{\partial}{\partial_{r}}\left[\frac{1}{r} \frac{\partial}{\partial r} r \frac{\partial \psi}{\partial r}\right]
$$

where $\bar{\lambda}$ is an arbitrary scalar function closely related to our function $\Lambda(\Phi)$ of Eq. (11), and $\Psi$ is the poloidal flux. In this I-D version we can compare our model to the Kadomtsev flux reconnection model [3].

\section{NUMERICAL METHOD}

We have developed a fully implicit code to implement our model in both 1-D and 2-D versions. In the 1-D version we advance the poloidal flux, $\Psi$, in time by solving Eq. (12). We normalize the equation to the resistive time. The value of the safety factor, $q$, can be found from the spatial derivative of $\Psi$ and the current density by differentiating once again. We solve Eq. (12) by using an implicit finite difference technique and reduce the equations to block tridiagonal form. We integrate $\Psi$ from the boundary inward with the boundary condition that the spatial derivative of $\Psi$ is constant at the edge. This corresponds to the condition of keeping the total integrated plasma current constant.

In the 1-D case we compare this model to the Kadomtsev reconnection. To calculate the Kadomtsev reconnection we let the poloidal flux evolve due to resistive relaxation using Eq. (12) with $\bar{\lambda}=0$. We convert to $m=1, n=1$ helical flux by subtracting the toroidal flux from the poloidal flux, then use the reconnection formulae given by Kadomtsev [3] to 
calculate the helical flux after reconnection. We then compare this to the effect of triggering the sawtooth by letting $\vec{\lambda}$ be nonzero in Eq. (12).

In the 2-D version we advance the inverse $q$ by solving Eq. (11) directly. We obtain the metric coefficients that appear in Eq. (11) by calling a 2-D MHD equilibrium program [15] that solves for the equilibrium, given the q-profile, at every iteration. The parallel current ciensity is then found from a derivative of the inverse $q$. The implicit finite differencing technique is similar to that used in the 1-D case, and the boundary condition is to keep $q(e d g$ s) constant. We again normalize the equation to the resistive time using the total toroidal flux divided by the toroidal field at the plasma edge as the characteristic square length.

To model sawteeth we begin with some initial $q$-profile with $q_{0} \approx 1$ and $q(r)$ increasing parabolically to the value at the outer radius, $q(e d g e)$. We let the current profile relax due to classical resistivity by solving Eyss. (11) or (12) with the hyper-resistivity term equal tc zero. When $q_{0}$ reaches a predetermined threshold, typically $q_{0}=0.9$, we allow the hyper-resistivity term to come on by changing $\lambda$ to a preselected nonzero form. The hyperresistivity term will have the effect of flattening the current and $q$ profiles in the region where $\lambda$ is nonzero. When the value of $q_{0}$ reaches $g_{0}=1.0$ we set the hyper-resistivity term back to zero, and allow the profiles to agajn relax due to classical resistivity only. We then repeat the entire process several times until a steady-state sartooth cycle is reached.

\section{TV. RESULTS}

In our 1-D cylindrical model we have found a form of the arbitrary functional coefficient, $\lambda$, with which our model ver. $j$ nearly matches the results of the Kadomtsev flux recennection model. We use a resistivity profile given by $\eta(r)=\eta_{0} \exp \left(4.5 r^{2}\right)$ corresponding to a resistivity that goes like $T^{-\frac{3}{2}}$ where the temperature profile is Gaussian. The hyper-resistivity profile is given by

$$
\lambda(r)=\left\{\begin{array}{cl}
\lambda_{0}\left(1-\left(\frac{r^{2}}{r_{*}^{2}}\right)\right)^{\alpha} & \text { for } r \leq r_{*} \\
0 & \text { for } r>r_{*} .
\end{array}\right.
$$

The value we use for $\lambda_{0}$ is 775 , and $\alpha$ is equal tu 1.20 . The value for $r_{\text {, }}$ is determined by finding the point where the $1 / 1$ helical flux is equal to the $1 / 1$ helical flux at the center. It is inside this point where the Kadomtsev reconnection takes place and that the hyperresistivity affects the flux. Outside of this point the flux is unchanged by both this moulel and the Kadomtsev model. Figure 1 shows the current profiles and q-profiles for both models immediately after triggering the sawtooth. Figure 2 shows how qo evolves in time according to both models. We see that for this form of $\lambda(\psi)$, the results of this model are nearly identical to those of the Kadomtsev model.

We can visualize how the current profile is affected by the hyper-resistivity as in Fig. 1. 
From the form of the Ohm's law, Eq. (1), we see that if the magnitude of $\lambda$ is large enough, then the hyper-resistivity term dominates the right-hand side of the equation. Furthermore, if $\lambda$ is large, then the gradient of $j_{\|} / B$ must become small in order for the right-hand side of Eq. (1) to balance with the left-hand side. In other words, $j_{1} / B$ becomes nearly uniform in the region where $\lambda(\psi)$ is large. Larger magnitudes of $\lambda$ will produce more uniform profiles of $j_{\|} / B$. Just outside the region where the current profile is flattened, we see a sharp current reversal. This corresponds to the region where $\lambda$ goes to zero. This negative current spike is a result of the inductive effect of the sudden mixing and equalizing of currents on adjacent magnetic surfaces [16]. In the region outside the current reversal the hyper-resistivity term is zero and has no effect on the equation. The profile is affected here only by classical resistivity; therefore the current profile in this region is essentially the same as it was before the sawtooth.

By choosing different magnitudes and functional forms for $\lambda$ we can model sawteeth that are quite different in their precise form, but with the same qualitative behavior as Kadomtsev sawteeth. The Kadomtsev model can thus be thought of as one specific case of a more general range of sawtooth behavior which can be represented by our model. For instance, by changing the position of the $\lambda$ cutoff, we can change the position of the current reversal and, therefore, determine how much of the inrier region of the current profile is flattened. By changing the magnitude of $\lambda$ we can specify how flat the resulting profile will be. If the magnitude is large enough, we can achieve a complete flattening of the current profile within this inner region. This is shown in Fig. 3. Once this limit in magnitude is reached, any further inerease in magnitude or change in the functional form of $\lambda$ will have no noticeable effect on the resulting profiles.

Figure 4 shows how the poloidal flux at the center, $\Psi_{0}$, evolves due to the effect of the sawteeth for both the Kadomtsev model and the hyper-resistivity model with $\lambda$ equal to that used in Fig. 1. This illustrates how the volt-seconds from the Ohmic coils are 'consumed' by the sawteeth in both models. The effect of sawteeth on volt-second consumption is of considerable importance to the operation of tokamaks during the current flat-top phase, especially for Compact Ignition Tokamak designs and for long-pulse reactors [5].

The above-mentioned functional forms of the hyper-resistivity are all such that $\lambda$ goes to zero exactly at some point and remains zero for all larger radi, i.e., these forms have a discontinuous derivative. Such a form is particularly well enited to match the Kadomtsev model, but may not be a good physical description of the hyper-resistivity. We can use forms of $\lambda$ that are continuous in all derivatives, and produce qualitatively similar results as long as the hyper-resistivity approaches zero fast enough with increasing radius. For example, forms such as $\lambda(r)=\lambda_{0} \exp \left(-a r^{p}\right)$ will produce behavior similar to the functional forms used above. The resulting current profile is not as flat and the current reversal is not nearly as sharp as in the Kadomtsev matching case, but the basic features are the same. Therefore, we know that it is not the discontinuity in $\lambda$ or its derivatives that determines the overall form of the profiles. Again, we have the freedom to change $\lambda_{0}, \alpha$, and $p$ in order 
to alter the precise form of the sawtooth behavior.

We extend our model to toroidal plasmas with cross-sectional shaping by using our full 2-D model. We use a form for $\lambda$ that corresponds to that which we used to match the Kadomtsev model in our 1-D version. This is, therefore, a 2-D exteasion of the Kadomtsev prescription to a toroidal plasma with a shaped cross section. We have run cases for plasmas with circular cross sections and compared the results with those for plasmas with highly shaped cross sections.

We consider two cases for our 2-D model: (a) a circular cross-sectional plasma and (b) a plasma with a strongly shaped cross section (elongation $=1.8$, and triangularity $=0.30$ ). Figure 5 shows the current density profiles ( $j_{\|} / B$ profiles) and the q-profiles for both cases at four times: at two times during the sawtooth crash, shortly after the sawtooth crash $\left(g_{0} \approx 1.0\right)$, and after some resistive relaxation and shortly before triggering the next sawtooth $\left(q_{0} \approx 0.91\right)$. The current profile of the elongated plasms evolves into a more hollow profile with the value on axis being significantly less than that at the peak. In fact, the value of the current on axis actually is higher during the first part of the sawtooth crash than it is just before the crash. This effect is much more pronounced for the elongated plasma than it is for the case with the circular cross section. To illustrate this, we have plotted the value of the current on axis, $j_{j}(0) / B$, versus time in Fig. 6. We see that there is a significant difference in the development of $j_{\|}(0) / B$ between the circular plasma and the elongated plasma. Figure 7 shows the corresponding time evolution of qo.

When the plasma recovers from a sawtooth crash, it begins with a fairly flat current profile. In the case of the highly shaped plasma, this current profile evolves into a slightly hollow profile during the initial resistive relaxation after the sawtooth. As this continues, the profile becomes more hollow, but the current that was forced outwards by the sawtooth begins to diffuse back towards the center. If this classical resistive diffusion continued indefinitely, the profile would, of course, evolve into its completely relaxed state, but the next sawtooth is triggered (at $q_{0} \approx 0.90$ ) while the profile is still quite hollow. As the sawtooth is triggered, the hyper-resistivity flattens the current profile, and the current density near the axis is larger than that before the sawtooth when there was a local minimum on axis due to the hollow current profile. Therefore, the sawtooth causes a sudden, roticeable jump in $j_{\mid f}(0) / B$. There is a corresponding drop in 90 . As the hyper-resistivity continues to flatten the current profile, nome of the current is redistributed towards the edge and the value of $j_{\|}(0) / B$ falls to the value it takes at the end of the crash. When the hyper-recistivity goes to zero at the end of the santooth crash, we have a nearly uniform current profile, and the whole process repeats itself. Keeping these steps in mind we can understand the evolution of $j_{\|}(0) / B$ in time as shown in Fig. 6(b). Furthermore, we can use the same reasoning to see why the evolution of $j_{\|}(0) / B$ in the case of the circular plasm, Fig, $6(\mathrm{a})$, looks so different.

The evolution of $j_{\|}(0) / B$ in the case of the circular plasma is not nearly so dramatic. 
The current profile at the time that the sawtooth is triggered (see Fig. 5(a)) is not nearly as hollow as in the case of the highly shaped plasma. Therefore, when the hyper-resistivity begins to flatten the current profile, $j_{\|}(0) / B$ experiences only a small jump, and then immediately drops as the current density becomes more uniform across the profile. After the crash the current diffuses back towards the center due to classical resistivity, and $j_{!}(0) / B$ increases again. Then the sawtooth is triggered again, and the whole process repeats itself. This gives a more conventional sawtooth pattern of $j_{\|}(0) / B$ for the circular plasma.

Figure 8 shows how the helicity, polojdal flux, and the total toroidal flux vary with time. We see that both the absolute and the relative change in the poioidal flux is substantially greater in the noncircular case, Fig. 8(b), than it is in the circular case, Fig. 8(a), even for the same relative change in $g_{0}$ during the sawtooth. The nearly discontinuous behavior of the poloidal flux in Figs. 8a,b is to be contrasted with the more gradual oscillations in the magnetic helicity, $K=\int_{0}^{\phi_{l}}\left(\Psi-\Psi_{l}\right) d \Phi$, where the subscript ' $l$ ' denotes the value at the limiter, of at the plasma edge. The helicity does not change discontinuously due to the direct action of the norzero $\lambda$ term, but does decrease due to the sharp current spikes created by the action of the sawtooth model. This decrease is compensated for, on the average, by the continued influx of helicity across the plasma boundary.

It is clear that all effects of the sawtooth oscillation are more pronounced in the noncircular cross-sectional geometry than in the circular cross section, although this result clearly depends on the assumption used here that the values of $q_{0}$ for which the sawtooth turns on and off are independent of the cross-sectional shape. The dominant effect of shaping the cross section is that for the same value of $q$ at the limiter, the radius over which the current rearrangement occurs is subatantially larger for the noncircular case than it is for the circular case. This is evident from comparing Figs. 5(a) and 5(b).

For cases with a larger value of $q$ at the limiter, $q(e d g e)$, the sawtooth affects a smaller region of the profile. This results in more conventional sawtooth patterns for $q_{0}$ and $j_{\|}(0) / B$ even for cases with highly shaped cross sections. Here, however, we have considered a case with low $q(e d g e)$ to demonstrate the strong effects of cross-sectional shaping on the sawtooth behavior.

\section{SUMMARY}

We have developed a fully implicit MHD transport code in 1-D and 2-D versions that models the effect of the sawtooth instability using a hyper-resistivity term. With the 1-D version we can closely match the results of the Kadomtsev model, by a suitable choice of our parameters determining the form of the hyper-resistjvity, $\lambda$. We can, given our freedom of this controlling function, also model sawteeth with behavior different from those of Kadomtsev. Thus, the Kadomtsev model may be regarded as a special case of the more 
general model discussed here. Furthermore, our model has been extended to plasmas with shaped cross sections through our $2-D$ version.

With the 2-D model we have found that plasmas with strong cross-sectional shaping develop hollow current profiles after the sawtooth crash and have a larger fraction of their area affected by the sawtooth region. The plasmas that are more strongly shaped develop profiles that are more hollow. The sawtooth crash will initially increase the current density near the axis before current is redistributed ontward by the hyper-resistivity. This results in an unusual sawtooth pattern for the current on axis, $j_{\|}(0) / B$.

Our form for the hyper-resistivity was chosen to be the form that matched the Kadomtsev model in the cylindrical limit, but it might be possible to develop a more self-consistent model by using a resistive stability code to calculate the eigenmodes and choose the form and magnitude of the controlling function, $\lambda(\psi)$, from the results of this stability analysis.

We have shown the utility of including a hyper-resistivity (or current viscoeity) term in an MHD transport code in order to model MHD phenomena such as the sawtooth instability. We have also seen evidence that such a term may be useful in the modeling of magnetic islands and tokamak disruptions, and in the modeling of dc helicity injection through induced double tearing modes as in the dc helicity injection scheme on CDX [17].

\section{ACKNOWLEDGMENTS}

This work was supported by U. S. DoE Contract DE-AC02-76-CHO-3073. One of the authors (DJW) is supported under the United States Air Force Laboratory Graduste Fellowship Program. 


\section{References}

[1] S. von Goeler, W. Stodiek, and N. Sauthoff, Phys. Rev. Lett. 33 (1974) 1201.

[2] B. Coppi, L. Sugiyama, Comm. Plasma Phys. and Controlled Fusion 10 (1986) 43.

[3] B. B. Kadomtsev, Sov. J. Plasma Physics 1 (1975) 389.

[4] S. C. Jardin et al., Nucl. Fusion 27 (1987) 569.

[5] W. A. Houlberg, Nucl. Fusion 27 (1987) 1009.

[6] J. Schmidt and S. Yoshikawa, Phys. Rev. Lett. 26 (1971) 753.

[7] T. H. Stix, Nucl. Fusion 18 (1978) 353.

[8] A. R. Jacobson and R. W. Moses, Phys. Rev. A 28 (1984) 3335.

[9] A. H. Boozer, J. Plasma Physics 35 (1986) 133.

[10] E. Hameiri and A. Bhattacharjec, Phys. Fluids 30 (1987) 1743.

[11] H. R. Strauss, Phys. Fluids 29 (1986) 3668.

[12] A. Bhattacharjee and E. Hameiri, Phys. Rev. Lett. 57 (1986) 206.

[13] S. C. Jardin, N. Pomphrey, and J. DeLucia, J. Comput. Phys. 68 (1986) 481.

[14] A. H. Boozer, Phys. Fluids 24 (1981) 1999.

[15] J. DeLucia, S. C. Jardin, and A. M. M. Todd, J. Comput. Phys. 37 (1980) 183.

[16] T. H. Stix, Phys. Rev. Lett. 36 (1976) 521.

[17] M. Ono et al., Phys. Rev. Lett. 58 (1987) 2165. 


\section{Figures}

Fig. 1. The current profiles and q-profiles for the Kadomtsev model and for the hyperresistivity model in the cylindrical limit.

Fig. 2. The time evolution of $q$ on axis, $q_{0}$, for both models.

Fig. 3. The current profiles and $q$-profiles for the Kadomtsev model and for the hyperresistivity model with a large value of $\lambda$, which gives complete flattening.

Fig. 4. The time evolution of the poloidal flux on axis, $\Psi_{0}$, for both models. Note that the curve for the Kadomtsev model has been displaced downwar, otherwise it would lie directly on top of the other curve.

Fig. 5. The current profiles and $q$-profiles at four different times during the sawtooth cycle for the 2-D model where we have (a) a circular plasma, (b) a highly shaped plasms (elongation $=1.8$, triangularity $=0.30$ ). The four times are shown on the time evolution of $q_{0}$ in Fig. 7. The radial variable $r$ is the square root of toroidal flux contained within the surface, normaiized to the value at the plasms edge.

Fig. 6. The time evolution of the current density on axis, $j_{\|}(0) / B$, for (a) the circular plasma and (b) the highly shaped plasma.

Fig. 7. The time evolution of the value of the safety factor on axis, $q_{0}$, for (a) the circular plasma and (b) the highly shaped plasma.

Fig. 8. The time evolution of the helicity, poloidal flux (ten times the difference between value at limiter and value on axis), and the total toroidal flux. 


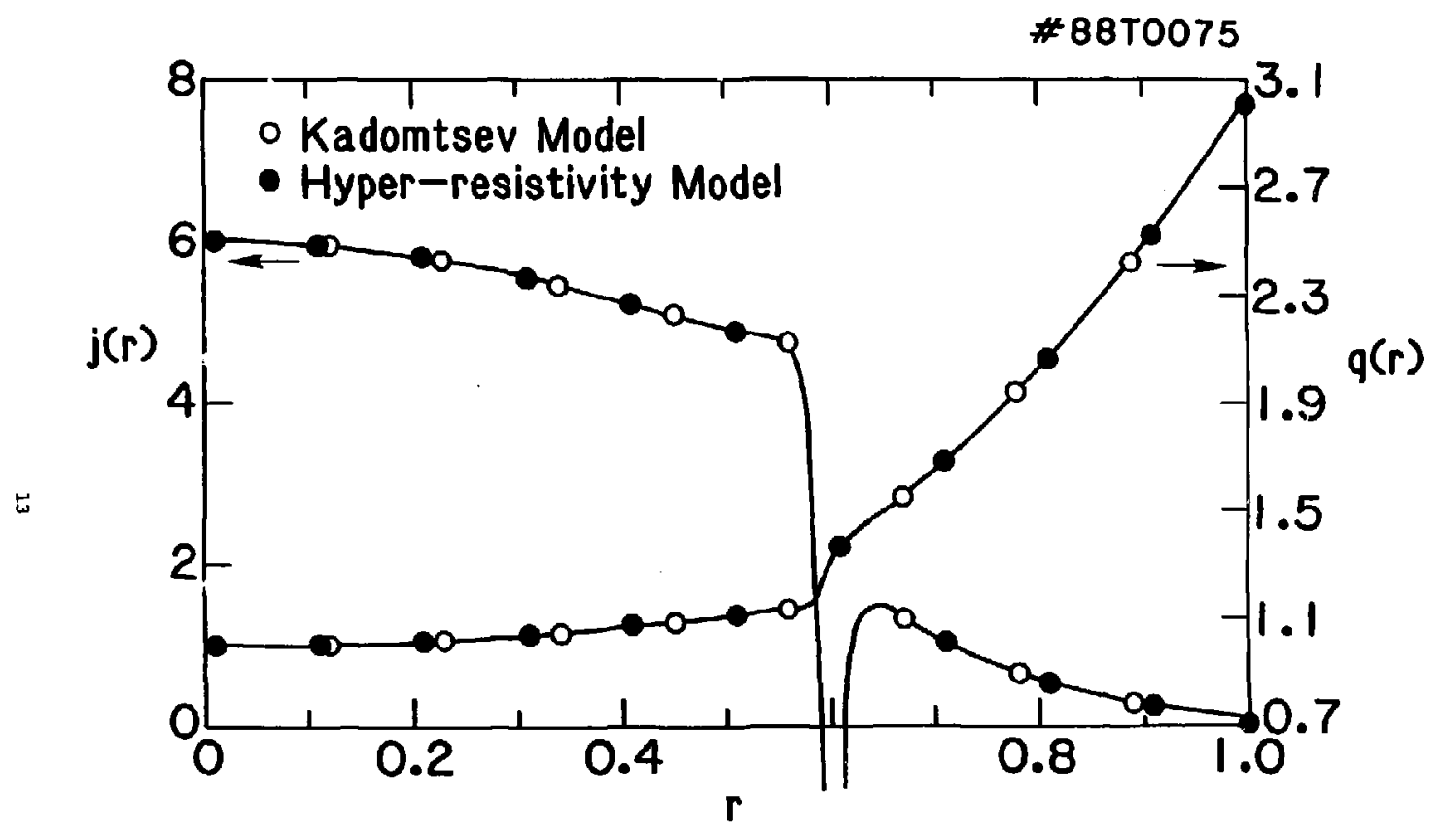

Fig. 1 


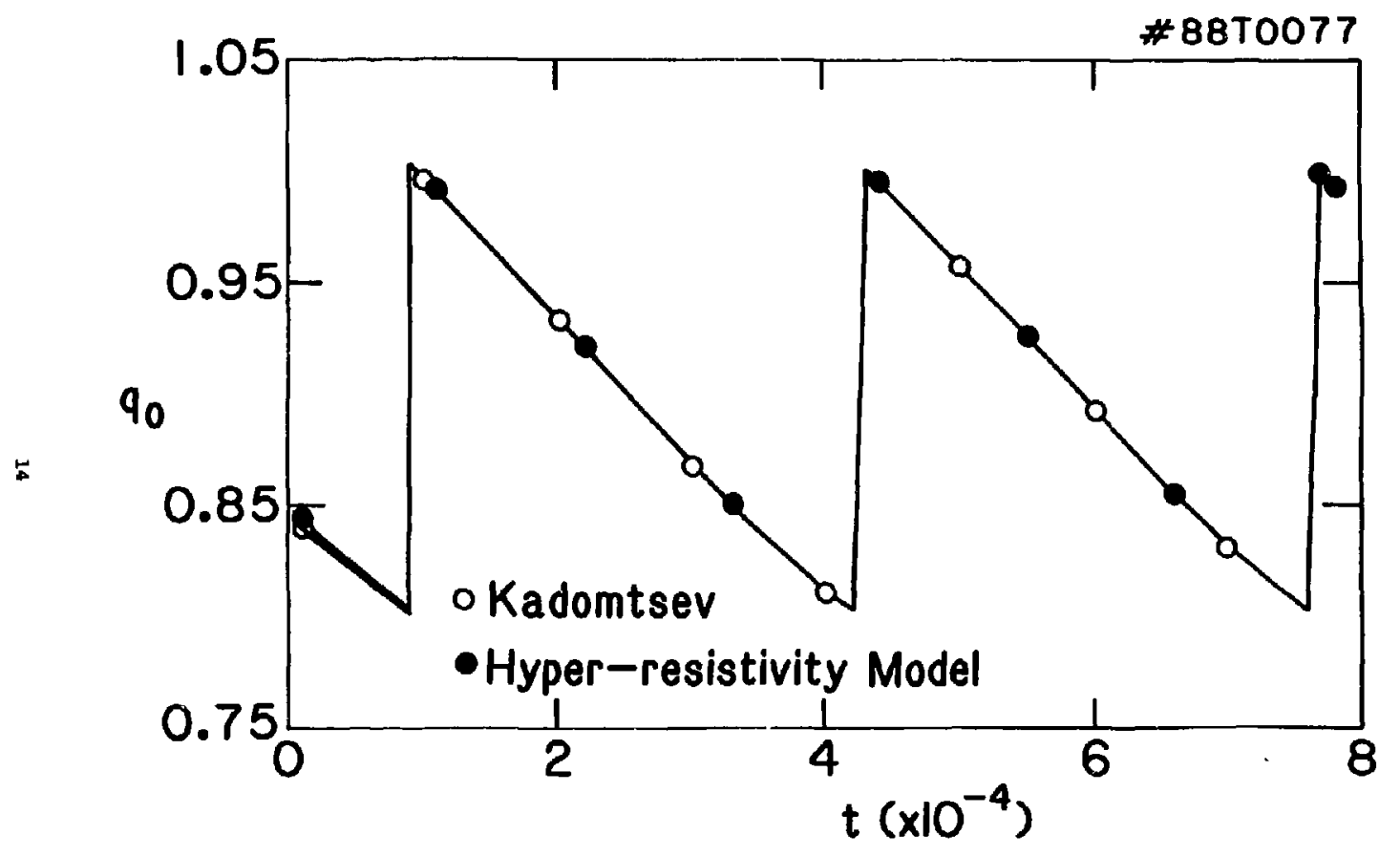

Fig. 2 


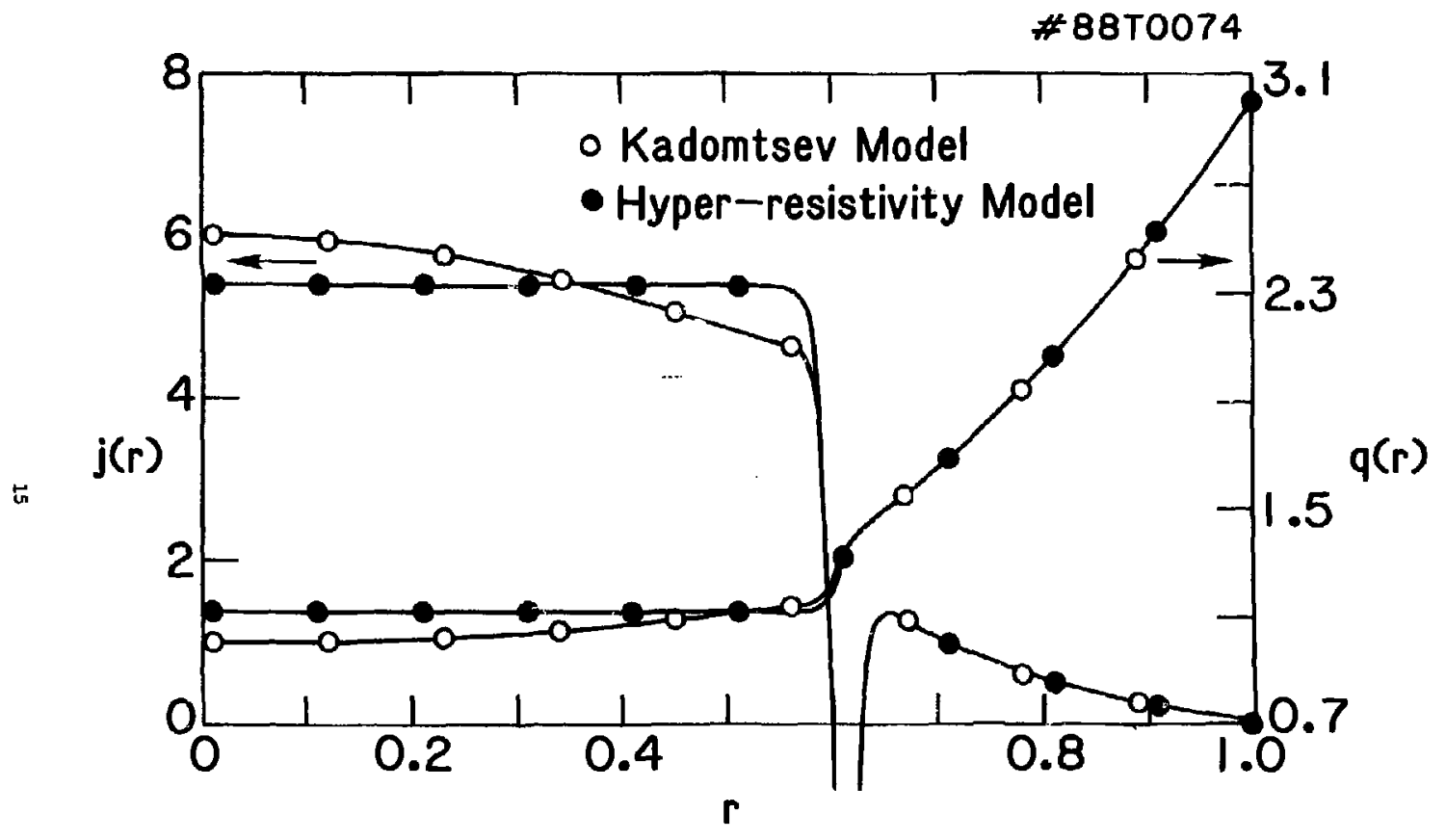




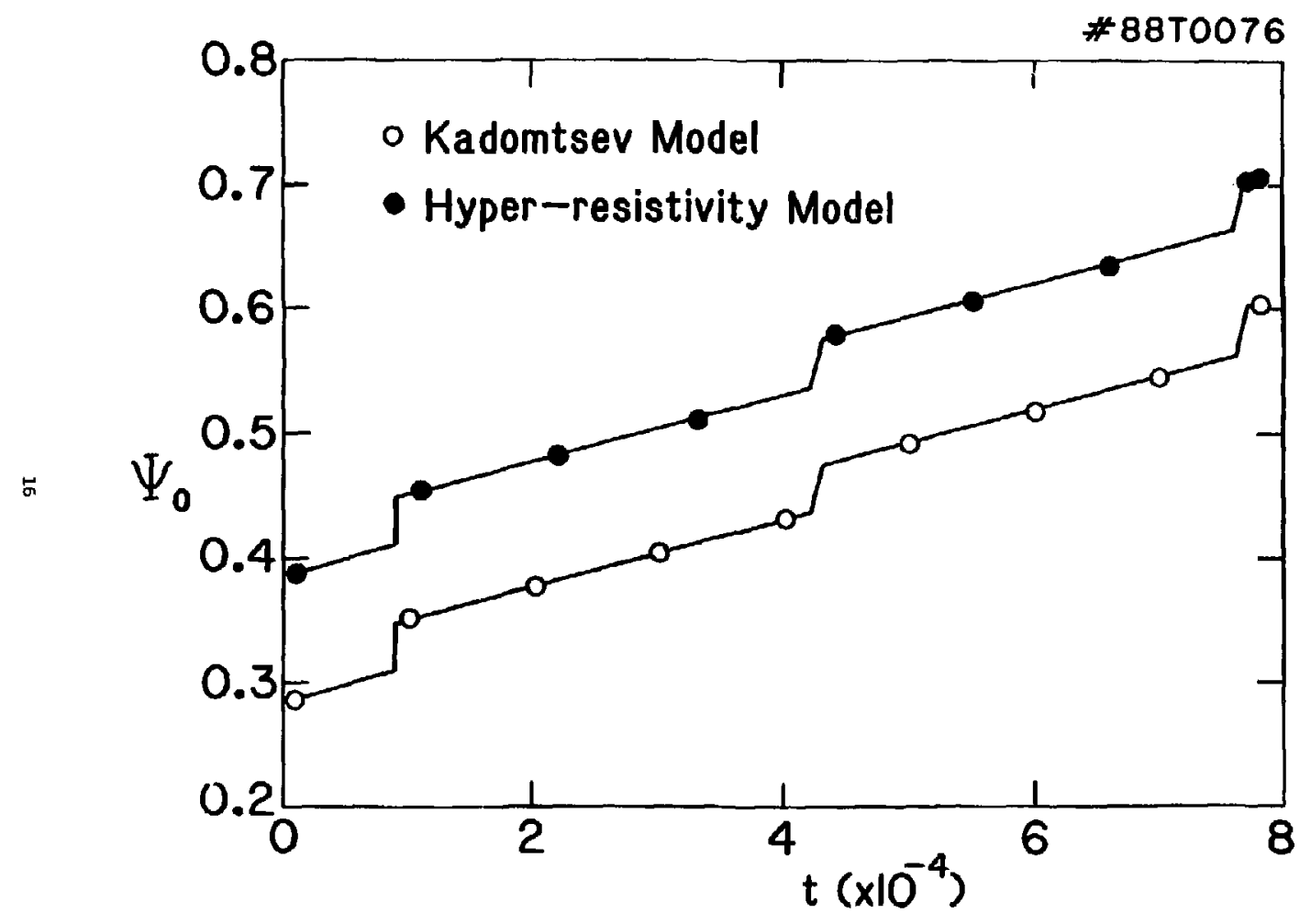

Fig. 4 
\#810078
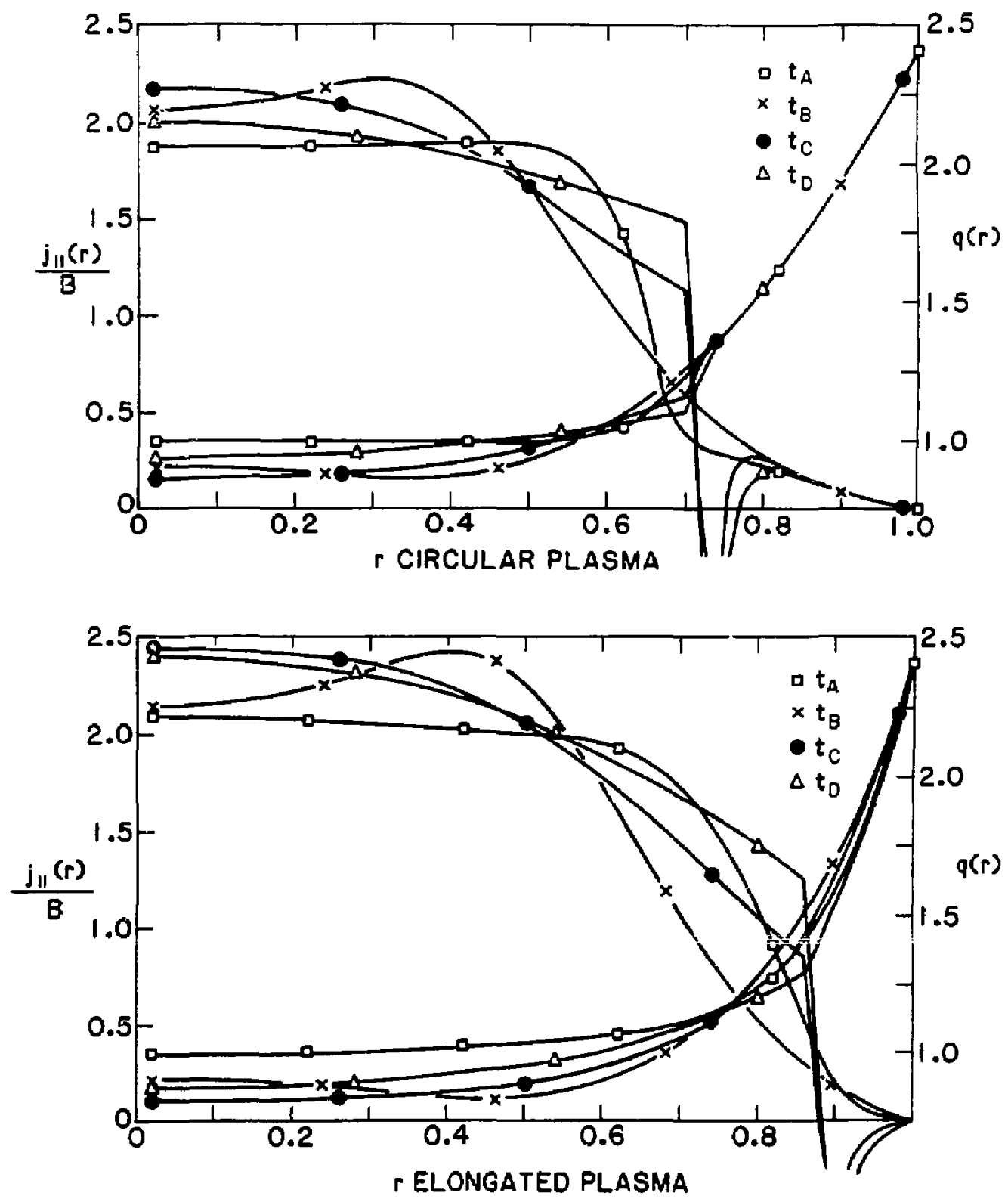

Fig. 5 

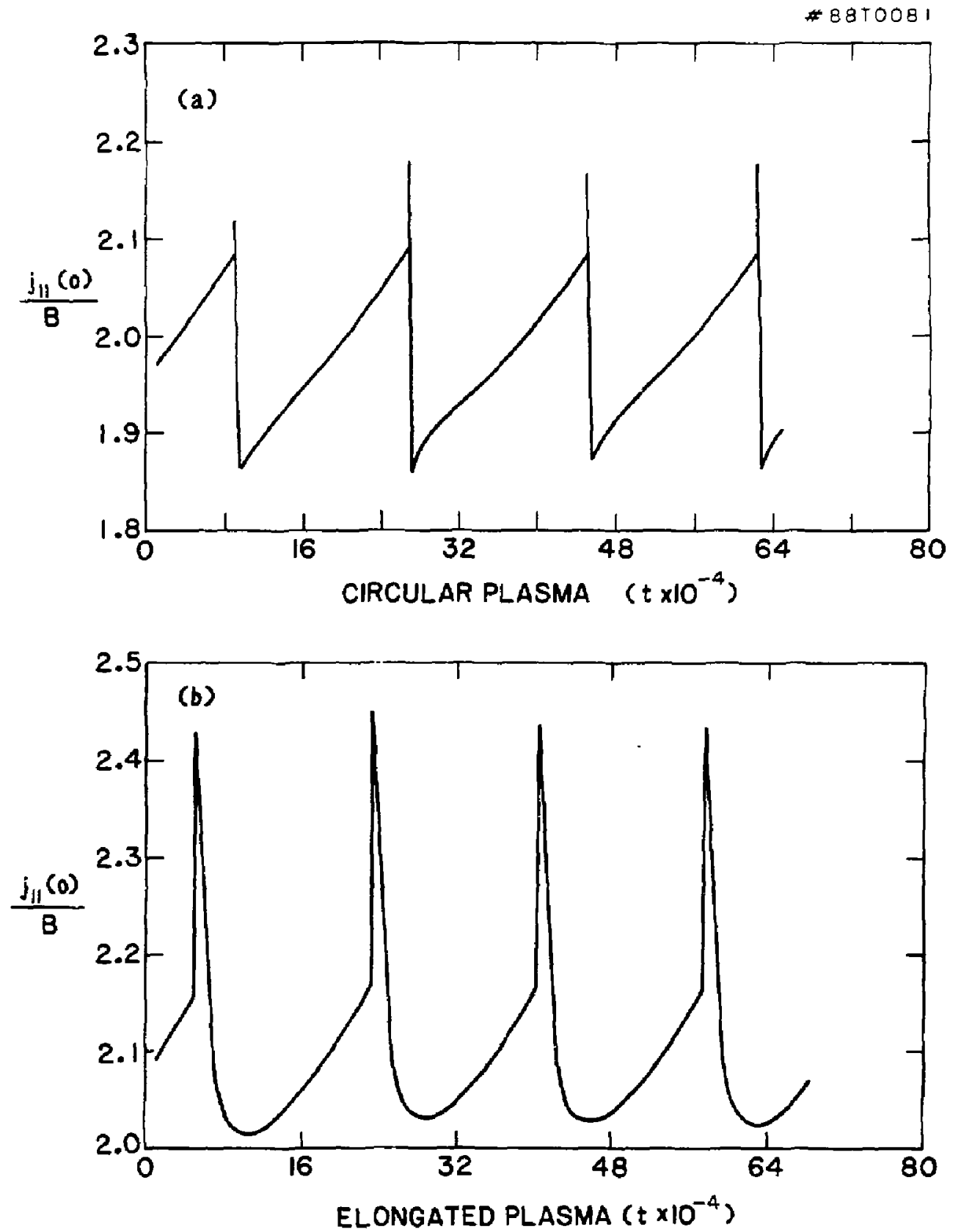

Fig. 6 

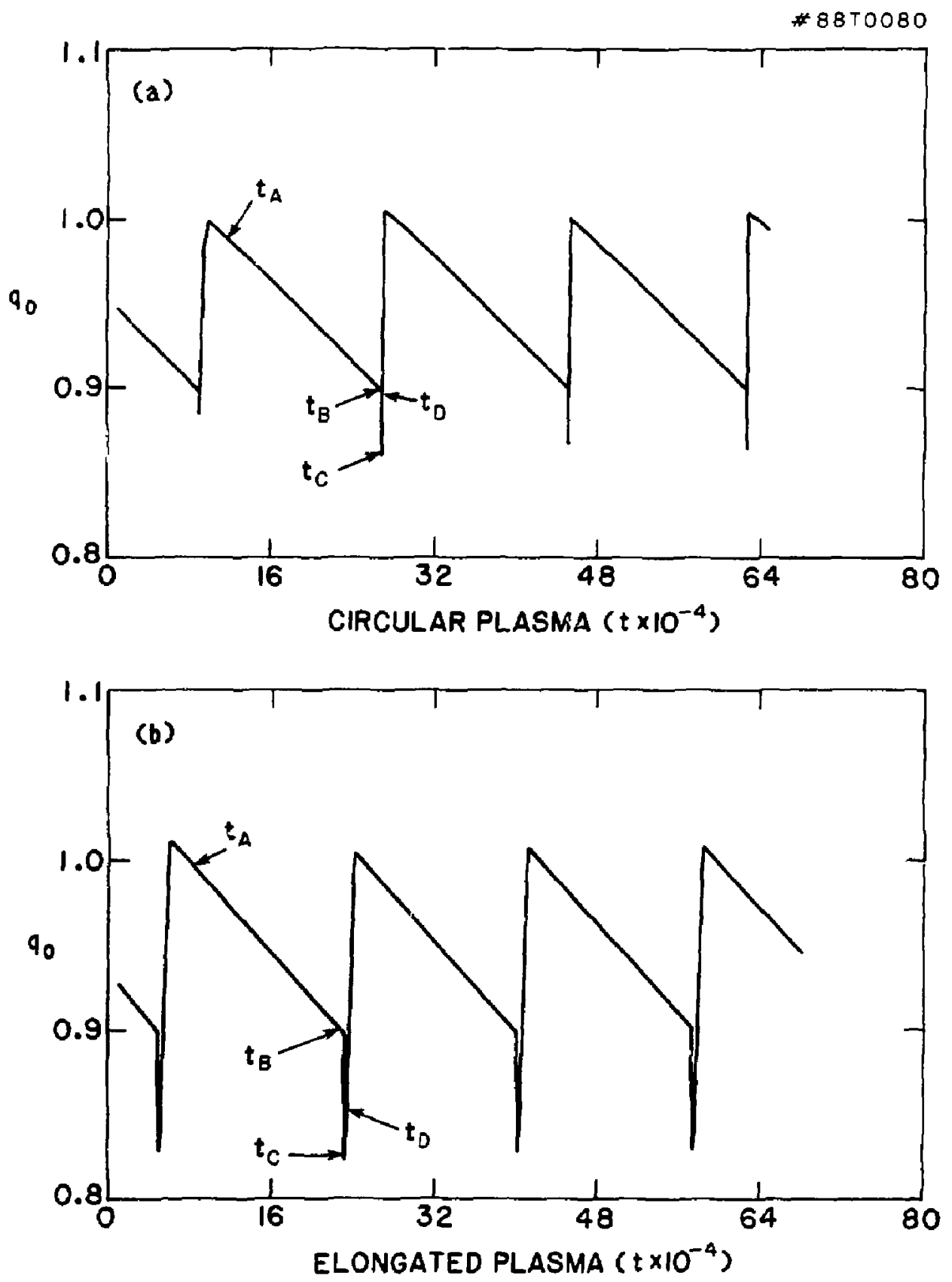

Fig. 7 

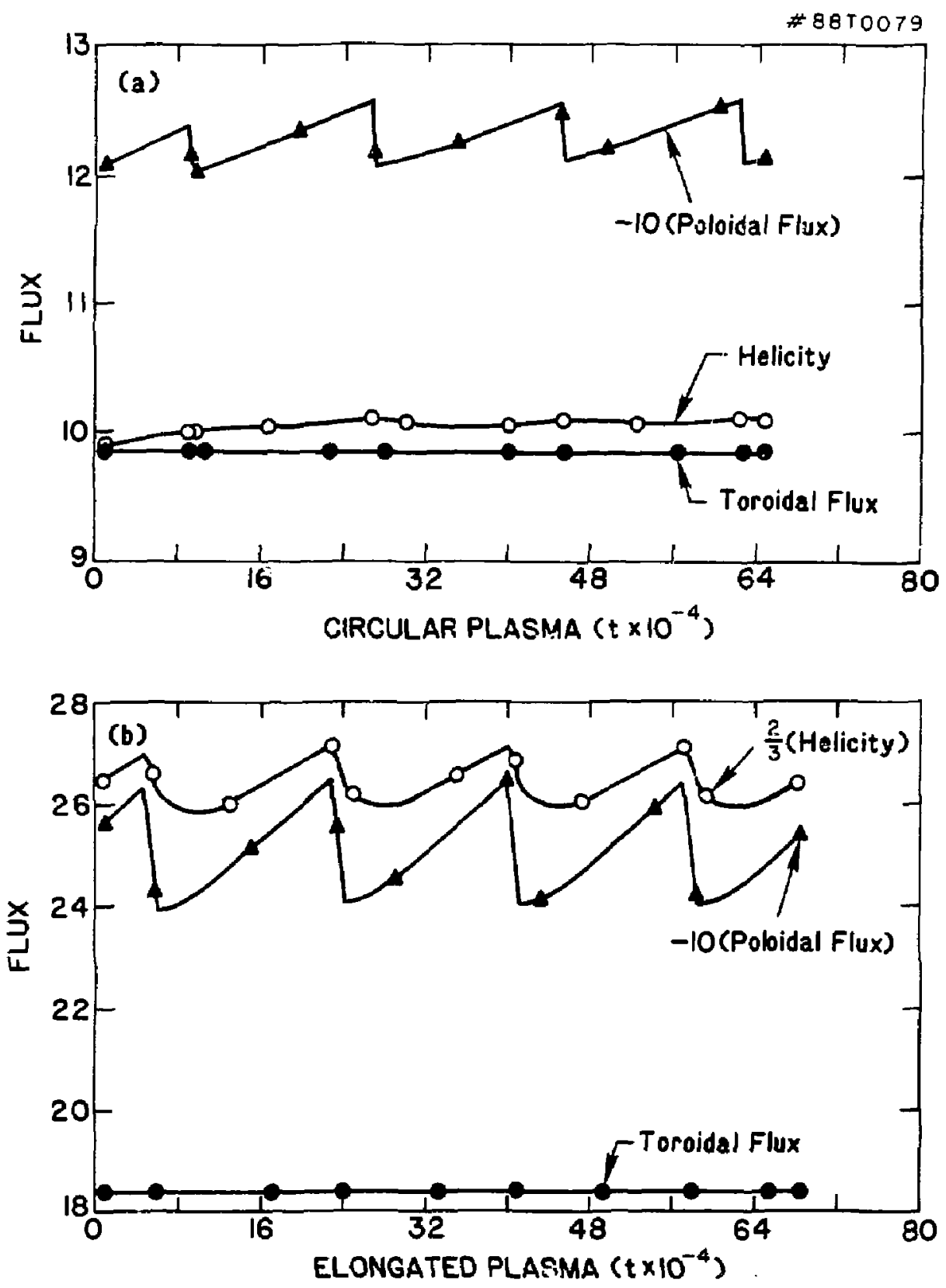

Fig. 8 
Or. Frank J. Pooloni, Univ of wollongong, AUSTRALIA Prof. M.H. Brennan, Univ Sydney, austrakiA Plasad Research Lab.. Australian Nat. Univ., AUSTRALIA Prof. I.R. Jones, Flinders Univ., AuSTRALIA Prof. F. Cap, Inst Thes Phys, AustRIA

Prof. M. Heinder, Institut fur Theoretische Physik, AUSTRIA H. Goossens, Astronomiseh Instituut, BELGILM Eeole Royale militaire, Lab de Phys PI asmas, BELgium Comission-Eurogaan, Dg-XII Fusion Prog, Belgium Prof. R. Boucique, Laboratoriun yoor Natuurkunde, BELGIUM Dr. P.H. Sakanaka, Instituto Fisica, BRAZIL Instituto Du Pesquisas Espaciasi-INPE, BRAZIL Documents Office, Atomic Energy of Canoda Limited, carkon Dr. M.P. Bachynski, wa Technologies, Ine., Canaba Or. H.M. Skarsgard, University of Saskatchoman, CNWDA Dr. H. Barnerd, University of Britlin Columbia, CNuDa Prof. J. Toichmenn, Univ. of Montreal, CAMOA Prot, S.R. Sreanivasan, University of Calgory, Cawon Prof. Tucor W. Johnston, INFS-Energle, CAwaA Dr. C.R, Janse, Univ, of Alberta, CAwäA Dr. Peter Lukoc, Komenskeho Univ, CzECHOSLOVAKIA The Libruriton, Culhe Laboratory, EkelANO The Librarion, Rutharford Appleton Laboratory, ENGLAD W. S,A. Hutchinson, SET Library, EkGLMD C. Mouttat, Lab. de Physique des Mll ieux lon Is és, France J. Redet, CEN/CADARACHE - Bat 506, FRANCE Univ. of losnnina, Library of Physics Dopt. GeECE Dr. Toa Mual, Academy Bibliographic Ser., hang kong Praprint Library, Hungarian Academy of Sciences, Huarar

Or. P. Kaw, Institute for Plasmo Reseureh, IMOIA Dr. Philio Rosenau, Israel Inst. Toch, ISRAEL Librarian, Int'I Ctr Theo Phys, ITALY Prol. G. Rostagni, Univ Ol Padova, ITAlr Miss Clelia O. Polo, Assoc Euentoh-ENEA, Itaky Biblioteca, Instituto al fisico del Plasm, ITALY Or. H. Yanoto, Toshlbe Res 8 Dov, JAPAY Prof. 1. Kowekel, Atealc Enorgy Res. Institute, JAPAH Prof. Kyaj I Nlshikwa, Univ of Hiroshide, JAPAN Direc. Oapt. Large Tokemax Ras. JAERI, JNPRA Prof, Satoshi Itoh, Kyuthu Unlversity, JAPAN Researeh Info Center, Megoya UnI versity, JAPAN Prof, S. Tonake, Kyoto University, JAPAN Library, Kyoto University, JAPAN Frof, Nobuyuki Inowe, University of Tokyo, JNPAN S. MOr $i$, JAERI, JAPAN Librarian, Kored Advanced Energy Ras, Institute, KopeA Prof. D.1. Choi, Aov. Inst Sel \& Teen, KOPEA Prof. 0.s. Liley, University of Weikato, NeW zEALANo Institute of PIasa Physics, PFople's RePuelic of CHIN Librarian, Institute of Phys., PECPLE's REPUELIC Of CHIM Library, Tsing Hua University, PEOPLE's REPUDLIC of CHIMA

2. Li, Southmost inst. Physics, PEOPLE'S REPU⿴LIC Of CHINA Prof. J.A.C. Cabral, Inst Superior Tecnico, PoRTugal Or. Octavian Patrus, AL I CuzA University, ROMANIA Dr. Jahan de Villiers, Fusion Studies, AEC, SO AFRicA Prof. M.A. Hel Ibarg, University of Hatal, SO AFRICA C.I.E.M.A.T., Fusion Div. Librory, SPAIN Dr. Lennart Stanflo, Univarsity of UMEA, SWEDEN LIbrary, Royal Inst Tech, SWEDEN Prot. Hans Wlinelinson, Chalmers Univ Tech, SWEDEN Contro Phys des Plasmas, Ecola Polytach Fed, SWITZERLANo BIbl lotheak, Fom-inst Voor Plasma-Fysica, THE NETHERLANDS Dr. D.D. Ayutor, Sibarian Acod Scl, USSR

Dr. G.A. El isuev, Kurchatov Institute, USSR

Dr. V,A, Glukhikh, Inst Electrophysical Apparatus, USSR

Dr. V.T. Tolok, Inst. Phys. Tech. USSR

Dr. L.M. Kourlzhnykh, Institute Gan. Physics, USSR Muclear Res. Establishennt, Julich LTA., W. GeraunY Bibliothek, inst. Fur Plasestorschung, W. Gefakinr Dr. K. Sehindler, Ruhr Universitat Boenum, W. GEPunY ASOEX Rosding Ru, IPP/Max-Pl anck-Institut fur Plasmaphysik, W. GERMawr LIbrarlan, Max-PIanck Institut, W. ERPuHr Prof. R.K. Janev, Inst Pnys, rucoslavia 